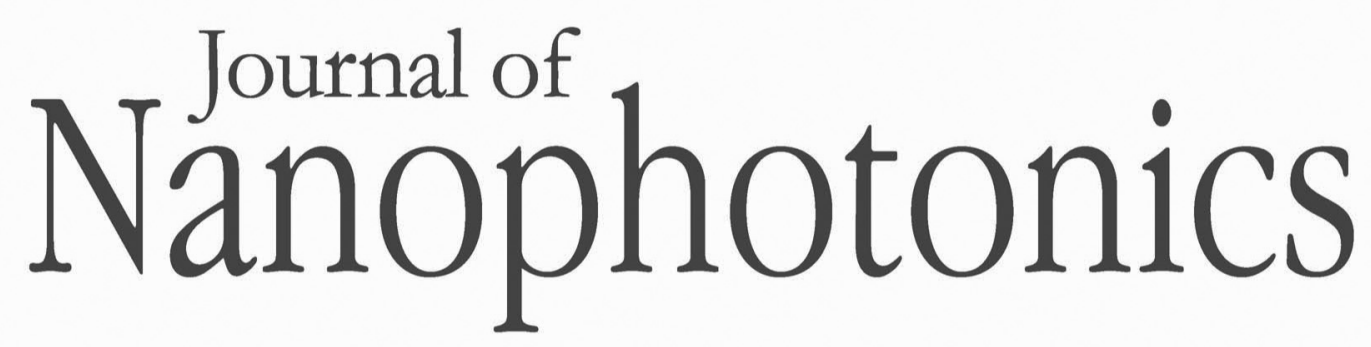

\title{
Optical characterization of refractive index sensors based on planar waveguide Fabry-Pérot Bragg grating cavity
}

Sang-Mae Lee

Won-Taek Jeong

Kyung-Chun Kim

Kyung-Jo Kim

Mincheol Oh

Simarjeet S. Saini

Mario Dagenais

\section{O SPIE}




\title{
Optical characterization of refractive index sensors based on planar waveguide Fabry-Pérot Bragg grating cavity
}

\author{
Sang-Mae Lee, ${ }^{\text {a }}$ Won-Taek Jeong, ${ }^{\text {b }}$ Kyung-Chun Kim,,${ }^{\text {b Kyung-Jo Kim, }}$ \\ Mincheol Oh, ${ }^{d}$ Simarjeet S. Saini, ${ }^{e}$ and Mario Dagenais ${ }^{\mathrm{f}}$ \\ ${ }^{a}$ MEMS/NANO Fabrication Center, Busan Techno-Park, Pusan 609-735, Republic of Korea \\ smlee@btp.or.kr \\ ${ }^{b}$ Pusan National University, Department of Mechanical Engineering, Pusan 609-735, \\ Republic of Korea \\ 'University of Arizona, College of Optical Science, 1630 East University Boulevard, \\ Tucson, Arizona 85721 \\ ${ }^{\mathrm{d} P u s a n}$ National University, Department of Electrical Engineering, Pusan 609-735, \\ Republic of Korea \\ ${ }^{\mathrm{e}}$ University of Waterloo, Department of Electrical Engineering, Ontario N2L 3G1, Canada \\ ${ }^{\mathrm{f}}$ University of Maryland at College Park, Department of Electrical Engineering, Maryland 20742
}

\begin{abstract}
The evanescent field-based polymeric planar waveguide refractive index sensors having a high $Q$ Fabry-Pérot (FP) cavity between identical dual Bragg gratings corrugated on the surface of the planar waveguide were developed. The FP Bragg gratings cavity was fabricated with a cavity size of 5 and $7 \mathrm{~mm}$, respectively. The spectra of light reflected from fabricated Bragg gratings, which were butt joined, were measured and compared with different indices of surrounding media. It was obtained that the FP Bragg gratings cavity is more sensitive than the single Bragg grating. The sensor developed shows much promise in the application of biomedical diagnostics such as a biosensor and/or environmental monitoring systems. (C) The Authors. Published by SPIE under a Creative Commons Attribution 3.0 Unported License. Distribution or reproduction of this work in whole or in part requires full attribution of the original publication, including its DOI. [DOI: 10.1117/1.JNP.7.073792]
\end{abstract}

Keywords: polymeric planar waveguide; Bragg gratings; Fabry-Pérot cavity; refractive index; sensors; biosensors.

Paper 13044SSP received Jun. 20, 2013; revised manuscript received Nov. 4, 2013; accepted for publication Nov. 5, 2013; published online Dec. 16, 2013.

\section{Introduction}

Planar optical waveguide gratings have been used in variety of applications for optical components like couplers, beam splitters, modulators, filters, and sensors, etc. ${ }^{1,2}$ These devices are used to control lasers, spectrometers, and the measurement of the wavelength of light in a wide variety of fields. Since the 1990s, optical waveguide Bragg gratings have also been employed in optical sensors to measure both the mechanical and electrical conditions. This allows for the smaller footprint, lower weight, and simplification of measurement systems, in particular, because the resultant measurements can be encoded by Bragg resonance wavelength.

Authors have recently developed high sensitivity, high selectivity fiber Bragg grating sensors. ${ }^{3-8}$ For these sensors, we completely removed the cladding and even part of the core of the fiber. We have demonstrated sensors with sensitivity to changes of the index of refraction of order $7 \times 10^{-7}$. We typically etch our sensor down to a diameter of $5 \mu \mathrm{m}$ and can detect a wavelength change of $1 \mathrm{pm}$ or less in the center frequency of our Bragg grating sensor. We have used this sensor to detect monolayer deposition of small molecules of mass of order $250 \mathrm{Da}$. In particular, we have studied the real-time attachment of one monolayer of 3-Aminopropyltriethoxysilane (APTES) and 3-aminopropyl-methyl diethoxysilane (APMDS) on the sensor and have demonstrated the formation of a water layer on the sensor. We have also studied the covalent monolayer attachment of glucose to the sensor and the hybridization of DNA. 
The application of Bragg grating technology has expanded to other areas, such as temperature sensors based on the silica-based etched gratings ${ }^{9}$ reacting to thermo-optic effects. Yoon et al. ${ }^{10}$ fabricated a biopolymeric planar waveguide Bragg grating by synthesizing gelatin and chitosan that could be generated into optical materials having two different refractive indices to form an optical planar waveguide. Bragg grating technology has been expanding into other physical measurement applications for the potential devices to be used as diagnostic tools for biological and medical services. ${ }^{11}$

We extend this work by converting our fiber sensor to a planar single-mode ultra-high $Q$ microcavity sensor on polymeric waveguides by forming a single-mode planar Bragg grating Fabry-Pérot (FP) cavities. In order to realize high finesse planar Bragg grating FP cavities, we will have to develop the technology for fabricating two spatially separated identical gratings.

With regard to biosensors, label-free biodetection is possible by measuring the varying effective refractive index of the optical waveguide that occurs from biointeraction between antibodies/probe and antigen/target molecules. This takes place on the surface of the core layer of the optical waveguide. This occurs by means of the evanescent field penetrating the optical waveguide Bragg grating when light is incident on the optical waveguide Bragg grating. This sensor for biodetection can be made by carving the grating on the surface of the core layer of the optical waveguide to multiplex the Bragg grating wavelengths. Thus, the characteristics of Bragg grating sensors can be used to diagnose human diseases by detecting proteins in the human blood as biomarks. In addition, Bragg grating technology has the potential to be applied in a variety of other areas as well, such as the monitoring of food quality in manufacturing processes in the food industry, chemical and biological weapons-related measurement and detection activities in the military, and air and water quality measurements in the environmental protection industries.

The results from this investigation will also be used to develop a small sensor device with ultra-high sensitivity, which is achieved by forming an FP cavity between two Bragg gratings on the planar waveguide that has not been reported yet although an optical fiber FP Bragg grating cavity sensor has been developed. ${ }^{11}$ The FP Bragg grating cavity structure allows for the development of ultra-high sensitive sensors. The sensitivity of the sensor has the potential to be further improved by generating sharper resonance peaks than a single Bragg grating. Shifts in optical resonant peaks due to the change of optical path length between two identical gratings arise from the change in the effective refractive index of an FP Bragg grating cavity. In this investigation, this is caused by binding immobilized antibodies and antigens, which can then be detected and measured. In this study, FP Bragg grating cavity sensors will be designed, fabricated, and characterized as potential biosensors.

\section{Experiment}

Figure 1(a) illustrates a schematic of two identical gratings for forming the FP cavity. The length of a single Bragg grating inscribed on the single-mode core channel waveguide is $2.5 \mathrm{~mm}$. The length of the FP Bragg grating cavity formed by the identical dual Bragg grating is 5 and $7 \mathrm{~mm}$, respectively, to compare the effect of cavity length on sensitivity of a sensor by varying the cavity length.

For a planar waveguide grating, the center wavelength of the reflection of a grating, $\lambda_{B}$, is given $b^{9}$

$$
\lambda_{B}=2 n_{\mathrm{eff}} \lambda,
$$

where $\Lambda$ is the period of the Bragg grating determined by the two beam interference, and $n_{\text {eff }}$ is the effective refractive index of the waveguide. For the FP Bragg grating cavity formed by two identical planar waveguide Bragg gratings, we assume that the effective cavity length $L_{\mathrm{FP}}$ is constant and that the wavelength of a resonance mode, $\lambda_{R}$, must satisfy ${ }^{11}$

$$
m \lambda_{R} / 2=n_{\mathrm{eff}} L_{\mathrm{FP}}
$$




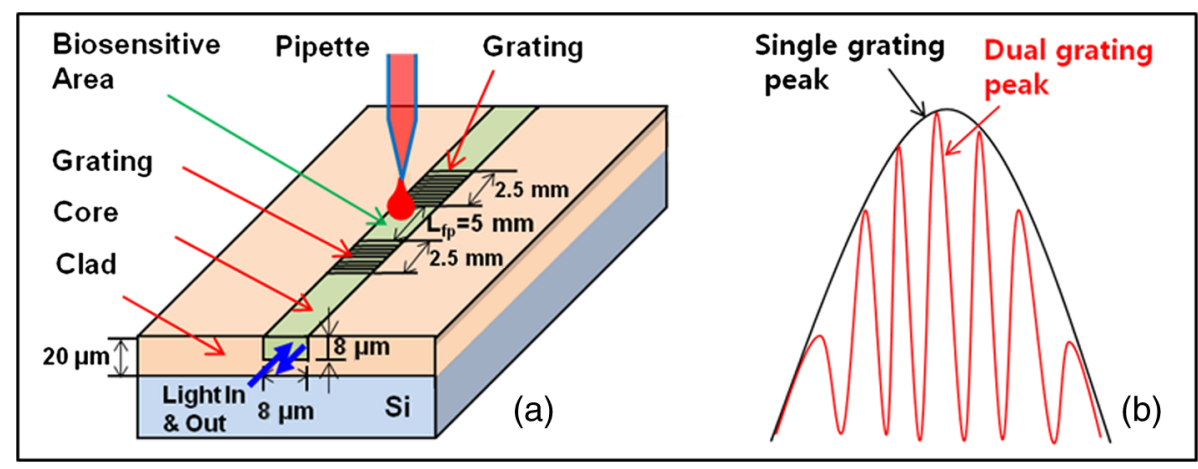

Fig. 1 Schematic of Fabry-Pérot (FP) Bragg grating cavity with 5-mm cavity (a) and its typical spectrum (b).

where $m$ is the modal number. When the refractive index of the ambient medium on the biosensitive area is changed, the effective index of the effective cavity length $L_{\mathrm{FP}}$ is changed and thus the resonance wave of $m$ 'th mode is shifted.

The period of the single grating was designed to be $538 \mathrm{~nm}$ so that the Bragg wavelength of the single grating should be obtained to be around $1550 \mathrm{~nm}$. The length of the single grating was $2.5 \mathrm{~mm}$, and the depth of the grating with a sine-waved pattern was designed to be $\sim 150 \mathrm{~nm}$ so that about $30 \%$ of the light can be reflected from the single grating.

Polymeric materials are used to form the planar optical waveguide which consists of the core layer (ZPU 13-440, $n=1.440$, Chemoptic, South Korea) and the cladding layer (ZPU 13-436, $n=1.436$, Chemoptic, South Korea). A cladding material, ZPU13-436, was spin coated on a 4 in. silicon wafer to be $20-\mu \mathrm{m}$ thick. After $3 \mathrm{~min}$ of UV curing under a H lamp with $9 \mathrm{~mW} / \mathrm{cm}^{2}$ at 370 -nm peak wavelength, the polymer was hard baked at $160^{\circ} \mathrm{C}$ for $30 \mathrm{~min}$. An optical waveguide pattern was defined on the cladding layer by conventional photolithography using an AZ5214 photoresist. After the cladding layer with a thickness of $20 \mu \mathrm{m}$ was deposited on the silicon substrate, the core channel groove of $8-\mu \mathrm{m}$ wide and $8-\mu \mathrm{m}$ high on the clad layer was made by a reactive ion etching to implement single-mode optical waveguide. The etch rate of the AZ5214 was slower than that of the ZPU polymer. Then Chemoptic's material, ZPU 13440 , was spin coated on the clad layer to form a core channel waveguide. The core layer was holographically exposed by two equal beams to form Bragg gratings on the core layer of the optical waveguide and then the exposed substrate was dry etched to form the corrugated gratings by the conventional lithographic technique, as shown in Fig. 2 .

To measure the shift in the optical Bragg grating wavelength when the FP cavity sensor is covered by fluids to be measured, erbium-doped fiber amplifier (LiComm, South Korea) and optical spectrum analyzer (ANDO, Japan) with a $0.05-\mathrm{nm}$ wavelength resolution were used. Fluids were dropped by a pipette on the surface of the cavity between the FP grating cavities because of measuring the fluid-induced change in optical path length of FP cavity.

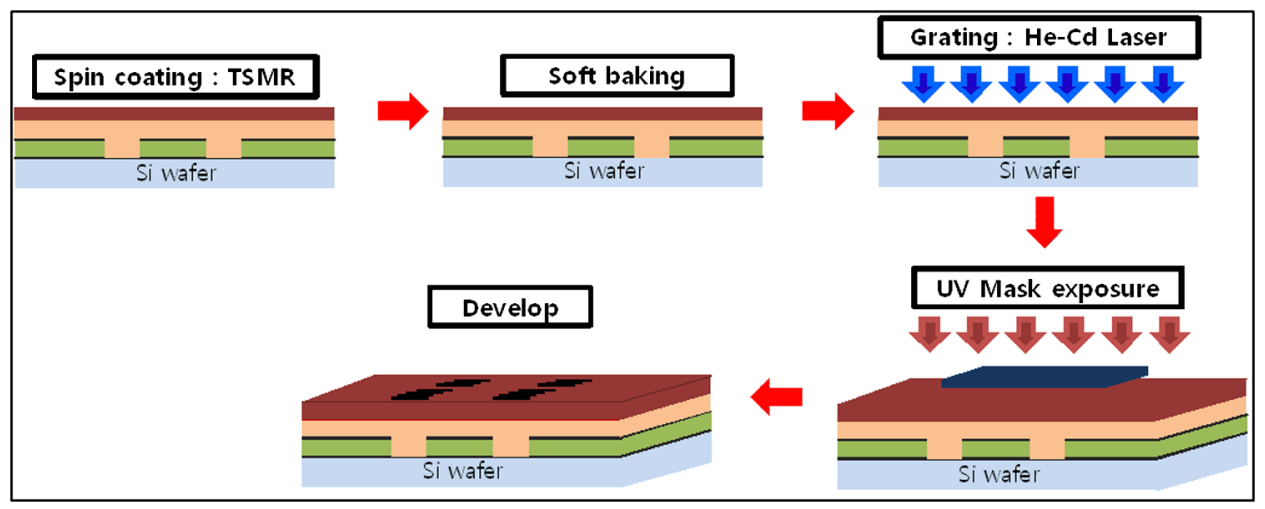

Fig. 2 Schematic of fabrication procedures for FP Bragg grating cavity. 


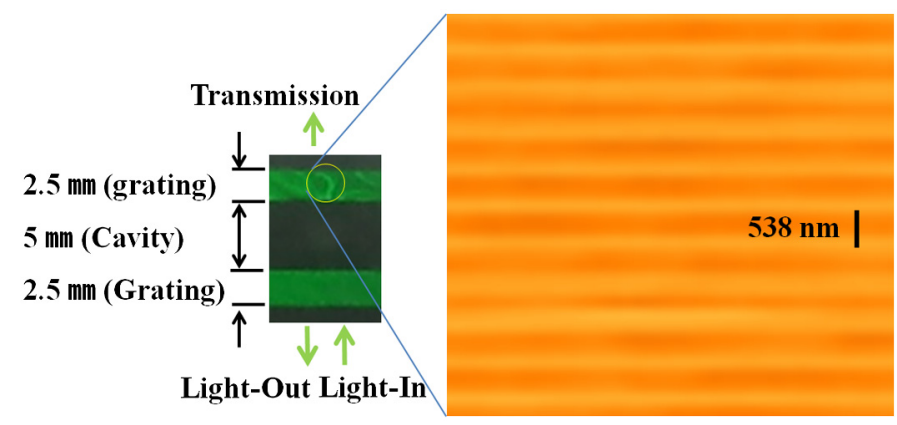

Fig. 3 Fabricated FP grating cavity and its AFM image.

\section{Results}

Figure 3 shows the photo of the fabricated FP cavity sensor. Atomic Force Microscopy (AFM) image of Bragg grating was also shown with magnification. The length of the FP cavity $\left(L_{\mathrm{fp}}\right)$ formed by the two identical Bragg gratings is $5 \mathrm{~mm}$. The reflected spectral resonance was measured by coupling the reflected light from the Bragg grating cavity to the detector through the $2 \times 1$ optical fiber coupler. Before fabricating the $2.5-\mathrm{mm}$ grating, the $5-\mathrm{mm}$ grating was fabricated as a preliminary experiment. However, it was found that the long-length grating caused significant reflection of light from the first Bragg grating and thus the interference between two gratings could not be made without sufficient light transmission from the first grating. To overcome this problem, the Bragg grating length was designed to be $2.5 \mathrm{~mm}$, preventing too much back-reflection from the first grating and thus transmitting 60\% to $70 \%$ of light from the first Bragg grating to the second Bragg grating. After passing the first Bragg grating, the light travels to the second Bragg grating and is then reflected to the first grating enough to have interference between two gratings, obtaining a high reflectivity from the FP cavity. The measured spectra of the fabricated FP Bragg grating cavity sensor were shown in Fig. 4.

The full-width at half maximum (FWHM) of the highest peak of spectrum formed by multiple reflection arisen from Bragg grating FP cavity was measured to be $0.036 \mathrm{~nm}$ with maximum reflectivity of $20 \mathrm{~dB}$ at wavelength of $1547.9 \mathrm{~nm}$, whereas that of the single grating was $0.228 \mathrm{~nm}$. Free spectral range of FP cavity was measured to be about $0.12 \mathrm{~nm}$, which was made by interference between the optical waves from the first Bragg grating and the second Bragg grating. In addition, the spectrum was formed symmetrically around the highest peak, as shown in Fig. 4.

To measure the sensitivity of the sensor, shifts in the wavelength of the FP cavity sensor were measured when dropping water and isopropyl alcohol (IPA) on the region (so called the biosensitive area) between the two Bragg gratings. At this moment, no surrounding liquid was dropped on the surface of the grating. A shift in wavelength of spectral resonant peak can be induced by changing the effective refractive index of optical waveguide due to varying the surrounding medium.

Figure 5 shows the spectral change of a single Bragg grating. Each spectrum was measured in air $(n=1.00)$, water $(n=1.33)$, and IPA $(n=1.37)$. The shifted wavelength was measured to
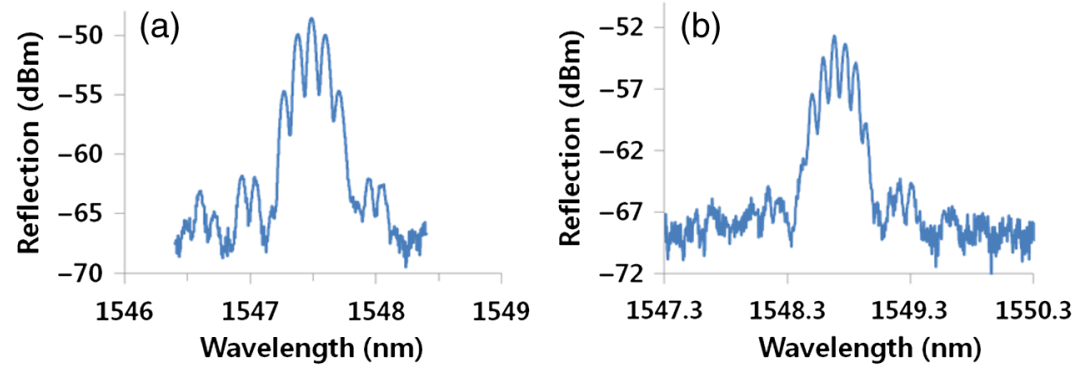

Fig. 4 Measured spectrum of Bragg grating FP cavity formed on planar waveguide (a) 5-mm cavity length and (b) 7-mm cavity length. 


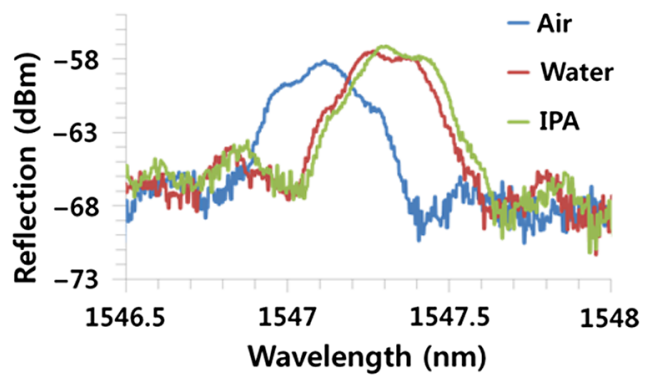

Fig. 5 Shift in wavelength of single-mode Bragg grating.

be $1547.099,1547.307$, and $1547.341 \mathrm{~nm}$ in air, when water was dropped on the single grating, and in IPA, respectively. Water- and IPA-induced shifts in the wavelength to the right were measured to be $\sim 0.208$ and $0.242 \mathrm{~nm}$, respectively. The difference in wavelength between water- and IPA-induced shifts was $0.034 \mathrm{~nm}$. The sensitivity of a sensor $(\Delta \lambda / \Delta n)$, i.e., wavelength on the refractive index unit (RIU) between air and water, was $0.63 \mathrm{~nm} / \mathrm{RIU}$. The sensitivity between water and IPA was $0.85 \mathrm{~nm} / \mathrm{RIU}$.

Figure 6(a) shows the spectral shifts of the FP cavity sensor with a 5-mm cavity length. The wavelength at the highest spectral reflectance was measured to be $1547.251 \mathrm{~nm}$ when the sensor was soaked in air. A shift in wavelength was measured to be $1547.411 \mathrm{~nm}$ when water was dropped in the cavity and $1547.513 \mathrm{~nm}$ at IPA. Water- and IPA-induced shifts in wavelength to the right by $\sim 0.16$ and $0.263 \mathrm{~nm}$ were measured, respectively. The difference in wavelength between water- and IPA-induced shifts was $0.103 \mathrm{~nm}$. The sensitivity of a sensor $(\Delta \lambda / \Delta n)$, i.e., wavelength on the RIU between air and water, was $0.48 \mathrm{~nm} / \mathrm{RIU}$. The sensitivity between water and IPA was $2.58 \mathrm{~nm} / \mathrm{RIU}$. It is shown that the closer the refractive index of the medium to that of the waveguide, the larger the movement of the peak. This phenomenon is more dominant for an FP cavity than a single grating.

Figure 6(b) shows the spectral shifts of the FP cavity sensor with $7 \mathrm{~mm}$ of a cavity length. The shifted wavelength at the highest value for each spectral reflectance was $1548.7 \mathrm{~nm}$ at air, $1548.9 \mathrm{~nm}$ at water, and $1549.0 \mathrm{~nm}$ at IPA. A water-induced shift in wavelength was $0.2 \mathrm{~nm}$, and IPA-induced shift in wavelength was $0.3 \mathrm{~nm}$. The difference in water- and IPA-induced shifts in wavelength was $0.1 \mathrm{~nm}$. The sensitivity of the sensor between air and water is $\sim 0.61 \mathrm{~nm} /$ RIU and that of the sensor between water and IPA is $\sim 2.5 \mathrm{~nm} /$ RIU.

Figure 7 shows the plots of shifts in wavelength of the Bragg grating sensor and FP Bragg grating cavity sensors with 5 and $7 \mathrm{~mm}$ length by air, water, and IPA. Due to the change in refractive index resulting from the change in the surrounding medium, the wavelength of the spectrum was shifted. The shifts in wavelength for the single Bragg grating and FP cavity sensors have a similar movement in water, but FP cavity sensors revealed more rapid movement than the single Bragg grating in IPA. Through these movements, when the refractive index of the surrounding medium approaches closer to the effective refractive index of the waveguide, the wavelength of the FP cavity sensors could be rapidly changed. The inset in Fig. 7
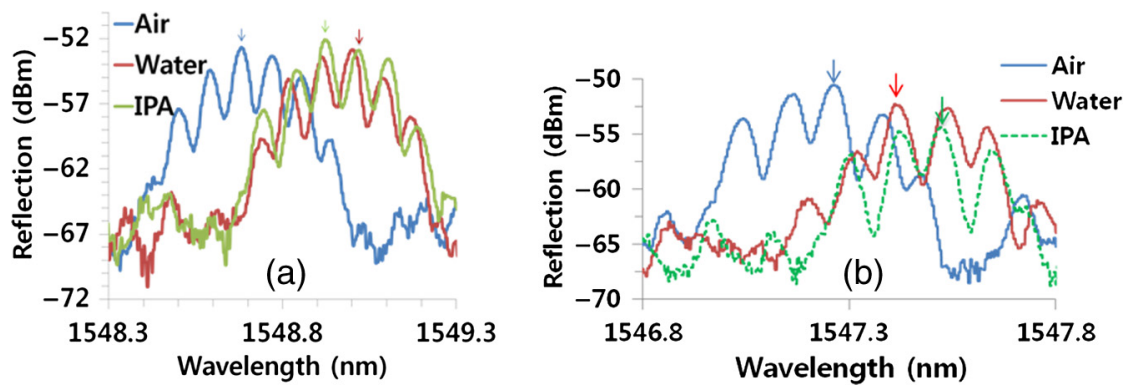

Fig. 6 Shift in wavelength of spectrum for FP Bragg grating with 5-mm cavity (a) and 7-mm cavity (b). 


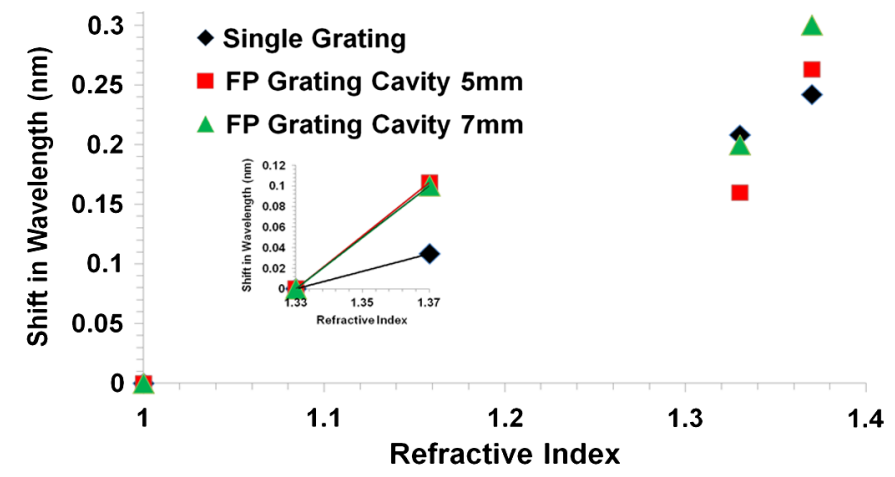

Fig. 7 Shift in wavelength of FP Bragg grating cavity sensor with low difference in refractive index between core and clad layers.

shows a comparison of shifts in resonance spectra for three sensors between water and IPA. FP Bragg grating cavity sensors revealed a rapid change of resonant wavelength compared to the single Bragg grating sensor. In addition, a shorter cavity length sensor showed more sensitivity than a longer cavity length sensor.

By comparison with the results of the sensor fabricated earlier, the planar waveguide with high difference in refractive index between the core and clad layers was made by using the ZPU 12-480 (core layer, $n=1.480$ ) and ZPU 13-436 (clad layer, $n=1.436$ ). Then the core layer was holographically exposed by two equal beams to form Bragg gratings on the core layer of the optical waveguide and the exposed substrate was dry etched to form the corrugated gratings by the conventional lithographic technique, as shown in Fig. 2 .

Figure 8 shows the spectral reflectance of the FP cavity sensor with $5 \mathrm{~mm}$ of length with a high difference refractive index between core and clad layers when surrounded by air, methanol, and water. The shifted wavelength at the highest value for each spectral reflectance was $1578.332 \mathrm{~nm}$ in air, $1579.24 \mathrm{~nm}$ in methanol, and $1579.321 \mathrm{~nm}$ in water, respectively. This result indicated a 10 times greater shift than low refractive difference cavity sensor by air and water, as compared with the results shown in Fig. 7. Figure 9 shows the plot of shifts in wavelength of FP Bragg grating cavity sensors by air $(n=1)$, methanol $(n=1.326)$, and water $(n=1.33)$. The curve of sensor sensitivity sharply increases between methanol and water because the refractive indices of surrounding media get close to that of the sensor.

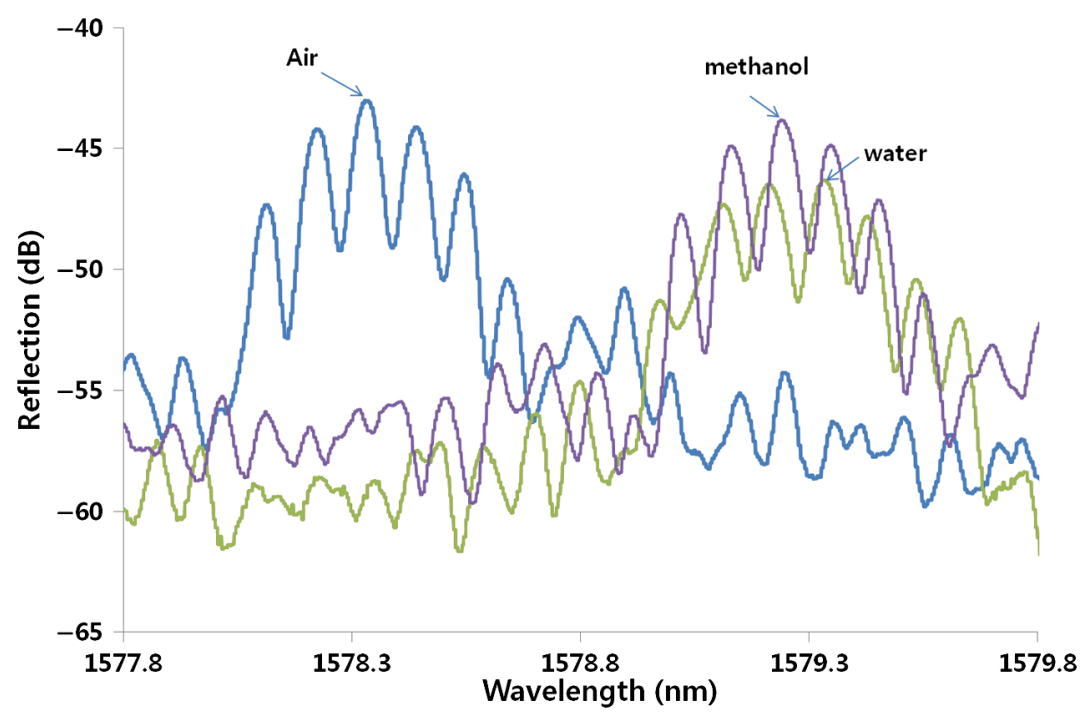

Fig. 8 Shift in wavelength of FP Bragg grating cavity sensor with high difference in refractive index between core and clad layers. 


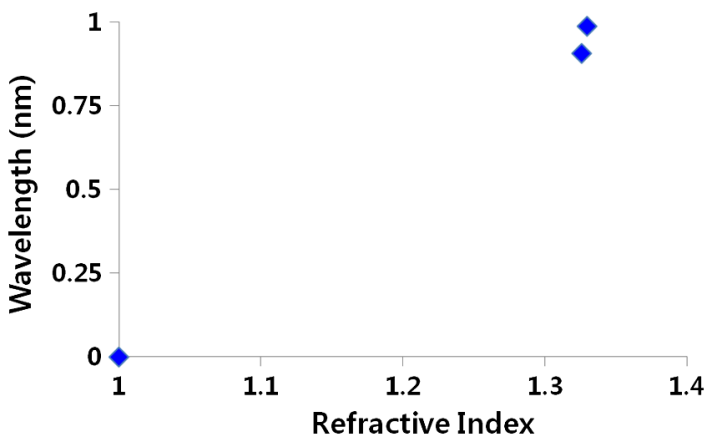

Fig. 9 Shift in wavelength of FP Bragg grating cavity sensor with planar waveguide with high difference in refractive index between the core and clad layers.

The FP Bragg grating cavity may have much better sensitivity than the single Bragg gratings. These results indicate that an FP Bragg grating cavity sensor may detect low volume of molecules with higher sensitivity as biological sensors when employing the immune-sensing schemes such as antibody and antigen reactions.

\section{Conclusion}

The purpose of this study is to develop biologically compatible high-sensitivity sensors for the detection of a biomedical substance for medical diagnostics through telemedicine via a bodymountable tiny small biochip and environmental monitoring. In this study, a polymeric optical planar waveguide was made by using Chemoptics company's ZPU13-436 for the cladding layer and ZPU13-440 for the core layer. As a preliminary study, we fabricated the sample which has a grating length of $5 \mathrm{~mm}$. As a result, it was confirmed that the shallower depth and the shorter grating length could develop the interference arisen from multiple reflections between two identical Bragg gratings.

We fabricated two single gratings to form a FP Bragg grating cavity that has 5 and $7 \mathrm{~mm}$ of cavity lengths. The Bragg wavelength of the single grating that forms the FP Bragg grating cavity was designed to be a 1550-nm wavelength.

As a result, spectral characteristics for the 5 and $7 \mathrm{~mm}$ of the Bragg grating cavity revealed a sharp-pointed peak that is finger shaped, whereas the single Bragg grating has a round-shaped peak. In addition, reflectivity of the single Bragg grating is about $10 \mathrm{~dB}$, but that of the 5 and $7 \mathrm{~mm}$ of Bragg grating cavities have about $20 \mathrm{~dB}$, which is two times larger than the single Bragg grating. Furthermore, the resonance spectra of the FP Bragg grating cavity sensors with the 5 and $7 \mathrm{~mm}$ Bragg grating cavity have 0.036 and $0.064 \mathrm{~nm}$ of FWHM as compared to $0.316 \mathrm{~nm}$ of FWHM for the single Bragg grating. This fact confirms that the resolution of the FP Bragg grating cavity sensor could be enhanced by 10 to 20 times over that of the single Bragg grating.

It is shown that the higher the refractive index of the medium, the more the resonance peak of a sensor moves. The Bragg grating cavity is more sensitive than the single Bragg grating at the same wavelength area. Therefore, a Bragg grating with a cavity area is more sensitive than the single Bragg grating. In addition, the shorter FP cavity waveguide could reveal much more sensitivity than the longer one.

\section{Acknowledgments}

This work was supported by the National Research Foundation Grant funded by the Korean Government (NRF-2010-6145). Mr. Won-Taek Jeong made Bragg gratings and optical coupling technique, Prof. Kyung-Chun Kim worked on data analysis, Dr. Kyung-Cho Kim fabricated the planar optical waveguide including core channel waveguide, Prof. Mincheol Oh designed a FP cavity, and Profs. Simarjeet Saini and Mario Dagenais provided the valuable suggestion and discussion through the entire process of the research. 


\section{References}

1. D. C. Flanders et al., "Grating filters for thin-film optical waveguides," Appl. Phys. Lett. 24(4), 194-196 (1974), http://dx.doi.org/10.1063/1.1655150.

2. C. S. Hong and J. B. Shellan, "Broad-band grating filters for thin-film optical waveguides," Appl. Phys. Lett. 31(4), 276-278 (1977), http://dx.doi.org/10.1063/1 .89660 .

3. A. N. Chryssis et al., "High sensitivity evanescent field fiber bragg grating sensor," IEEE Photonics Technol. Lett. 17(6), 1253-1255 (2005), http://dx.doi.org/10.1109/LPT.2005 .846953 .

4. A. Chryssis et al., "Increased sensitivity and parametric discrimination using higher order modes of etched-core fiber bragg grating sensors," IEEE Photonics Technol. Lett. 18(1), 178 (2006), http://dx.doi.org/10.1109/LPT.2005.861628.

5. S.S. Saini et al., "Monolayer detection of biochemical agents using etched-core fiber Bragg grating sensors," IEEE Photonics Technol. Lett. 19(18), 1341-1343 (2007), http://dx.doi .org/10.1109/LPT.2007.902345.

6. C. J. Stanford et al., "Real-time monitoring of siloxane monolayer film formation on silica using a fiber Bragg grating," Curr. Anal. Chem. 4(4), 356-361 (2008), http://dx.doi.org/10 $.2174 / 157341108785914916$.

7. M. Dagenais and C. J. Stanford, "Evanescent fiber Bragg grating bio-sensors," in VLSI Micro- and Nanophotonics: Science, Technology, and Applications, E.-H. Lee et al., Eds., Taylor and Francis, London, UK (2009).

8. C. J. Stanford et al., "Covalent attachment of carbohydrate derivatives to an evanescent wave fiber Bragg grating biosensor," J. Sensors 2009, 1-7, (2009), http://dx.doi.org/10 $.1155 / 2009 / 982658$.

9. S. M. Lee, K. C. Ahn, and J. S. Sirkis, "Planar optical waveguide temperature sensor based on etched Bragg gratings considering nonlinear thermo-optic effect," KSME Int. J. 15(3), 309-319 (2001), http://dx.doi.org/10.1007/BF03185214.

10. S. H. Yoon et al., "Development of the biopolymeric optical planar waveguide with nanopattern," J. Surf. Eng. Mater. Adv. Technol. 01(02), 56-61 (2011), http://dx.doi.org/10 .4236/jsemat.2011.12009.

11. W. Liang et al., "Highly sensitive fiber Bragg grating refractive index sensors," Appl. Phys. Lett. 86(15), 151122 (2005), http://dx.doi.org/10.1063/1.1904716.

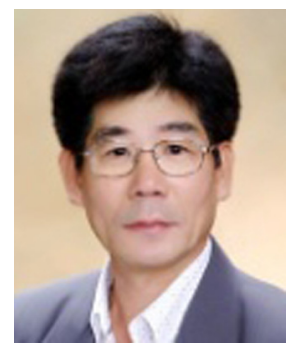

Sang-Mae Lee received his $\mathrm{PhD}$ in 1987 from the Department of Mechanical Engineering, Pusan National University, Pusan, South Korea. He was a professor of Yonam College of Engineering, Chinju, South Korea from 1988 to 1995. From 1995 to 1998, he was a research faculty at the University of Maryland College Park, Maryland, where he developed optical fiber and planar waveguide grating sensors. He also developed the MEMS sensor at the University of Cincinnati, Cincinnati, Ohio, from 1999 to 2001. Again, he joined the Department of Electrical and Computer Engineering at the University of Maryland College Park, Maryland, where he develop fiber Bragg grating sensors. After he returned to Korea, he served as a professor at Pusan National University until 2011. He is currently a director for MEMS/Nano Fabrication Center, Busan Techno-Park, Busan, South Korea. His research interest focuses on MEMS sensors and optofluidic sensor.

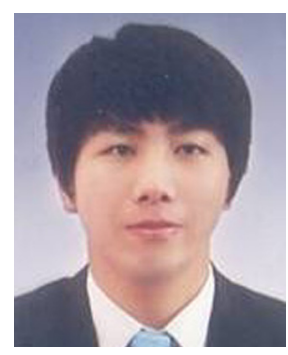

Won-Taek Jeong earned his BS from the Department of Mechanical Engineering, Dong-A University, Pusan, South Korea, in 2009 and MS from the Department of Mechanical Engineering Pusan National University, Pusan, South Korea, in 2011. 


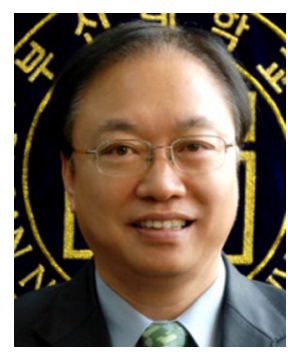

Kyung Chun Kim received his $\mathrm{PhD}$ degree at the Department of Mechanical Engineering of KAIST, Korea, in 1987. He received the Nakayama award (2013) from ASV12. He established the MEMS/ NANO Fabrication Center and served as the director from 2004 to 2009. In 2004, he was selected as a member of National Academy of Engineering of Korea. From 2008 to 2010, he was the president of the Korean Society of Visualization. In 2010, he was the chairman of the 14th International Symposium on Flow Visualization. Since 2005, he has been invited more than 10 times as a plenary speaker at reputable international conferences such as ISFV, PSFVIP, ISPIV, ISALTFM, ASV, etc. Currently, he is an editor-in-chief of the Journal of Flow Visualization and Image Processing. His research interests include multi-phase flows in macro/micro scales, 3D3C micro-PIV, micro/nano-scale energy transport, next generation marine engines, organic rankine cycle power generation, turbulence, and quantitative flow visualization (PIV, LIF).

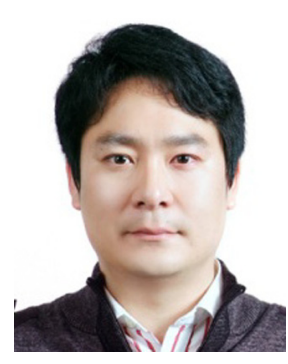

Kyung-Jo Kim received his BS, MS, and PhD degrees in electrical engineering from the Pusan National University (PNU), Busan, Korea, in 2006, 2008, and 2012, respectively. His thesis work at PNU was focused on optical sensors and widely wavelength-tunable lasers based on polymer waveguides. Since 2012, he has been a postdoctoral research associate in the College of Optical Science, University of Arizona, Tucson, Arizona. His current research interests include high-speed electro-optic polymer modulators, photonic integrated circuits, polymeric waveguide devices, and integrated optical sensors.

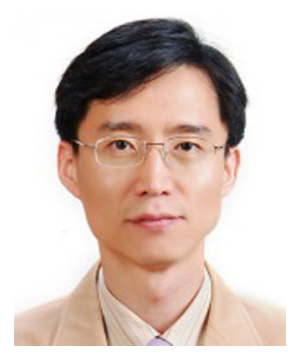

Min-Cheol Oh received his BS degree in the Communication Engineering Department of Korea Advanced Institute of Science and Technology (KAIST) in 1990, and his MS and PhD degrees in electrical engineering from Electrical Engineering Department, KAIST, Korea, in 1992 and 1996, respectively. From 1996 to 1999, he was a senior research engineer at ETRI, Korea, where he had invented various novel polymeric waveguide devices. In 1999, he joined University of Southern California EE Department as a research associate to continue his involvement with polymer devices. From 2000 to 2002, he experienced directing two start-up companies, Pacific Wave Industries in the U.S. and Zenphotonics in Korea. Since 2003, he has been an associate professor of electronic engineering department in Pusan National University, Busan, Korea. His current research interests include integrated optical sensors, photonic integrated circuits, polymeric waveguide devices, biophotonic sensors, and organic electronic devices.

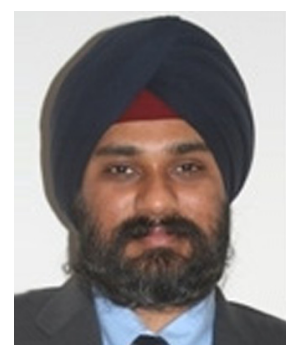

Simarjeet S. Saini is currently an assistant professor at the University of Waterloo. He did his BTech (Hons.) in electronics and electrical communication engineering from the Indian Institute of Technology, Kharagpur, India, in 1996 and $\mathrm{PhD}$ in electrical engineering from the University of Maryland, College Park, in 2001. His thesis research led to the formation of quantum photonics (currently Covega) in September 2000. From December 2000 to November 2004, he worked as a lead optoelectronics engineer at Covega Corporation, Jessup, Maryland, where he led the design and development of gain chips for external cavity lasers, semiconductor optical amplifiers, super-luminescent diodes, and high power lasers. In May 2004, he co-founded Altanet Communications to build fault tolerant ethernet WDM optical systems using intelligence in the optical layer. He is currently pursuing research in tunable quantum cascade lasers, biological and chemical sensors using fiber Bragg gratings and planar wave nanophotonic circuits, plasmionic lasers, all-optical packet networks using all-optical packet header recognition, and devices and networks for WDM-PON. He has over 60 publications in various peer reviewed journals and conferences, 5 U.S. patents granted and over 5 patents applied for. 


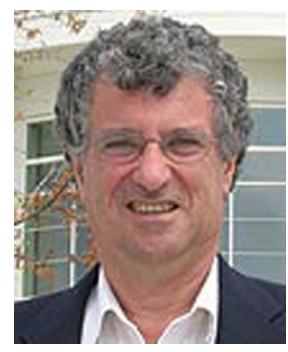

Mario Dagenais received his $\mathrm{PhD}$ from the University of Rochester in 1978. Together with Jeff Kimble, he made the first observation of photon antibunching. From 1980 to 1987, he worked at GTE Laboratories on photonic switching and semiconductor lasers. He joined the University of Maryland in 1987, where he has been professor of electrical and computer engineering. He has more than 300 archival and conference publications. He is VP membership for the Americas for the IEEE Photonics Society. $\mathrm{He}$ is a topical editor for the IEEE Photonics Journal. Recently, he has been interested in nanophotonics and plasmonics for the sub-wavelength confinement of light, in the direct rectification of visible light for collecting solar energy, in the development of interband cascade lasers and in glycobiology for the development of new, ultra-sensitive individual, and two-dimensional arrays of optical bio-sensors. 